\title{
The use of measuring phase-chronometric systems in the production of cyclic aggregates of aircraft
}

\author{
Ekaterina Metelkina ${ }^{1, *}$ and Aleksandr Komshin ${ }^{1}$ \\ ${ }^{1}$ Bauman MSTU, Machine building technology Department, 105005 Moscow, Russia
}

\begin{abstract}
The paper gives an assessment of the current technical condition of the machine-building industry as a whole in the aviation industry. Its weak sides are revealed. Based on the obtained data and the evaluation of existing methods for diagnosing cyclic mechanisms, an approach of the phase-chronometric method based on precise measurements of time intervals is proposed. A functional diagram of the information-measuring system is presented. The results of modeling the real and ideal operating cycle of the gear-boxes are analyzed.
\end{abstract}

\section{Introduction}

Wear of productive assets in Russia reaches critical values, but their renovation is rather slow. At the same time, the requirements to ensure their reliability only increase. In the park of operating machines and mechanisms, objects close to the exhaustion of their resources, hence potentially hazardous and requiring replacement, are preserved. Indicators reflecting the prospects for the use of a particular machine building object are determined by the level of working off of a design, manufacturing quality and informationmetrological support. [1]

Helicopter construction in the 21 st century is gaining momentum. The need for this type of air transport is obvious, due to its characteristics: maneuvering in urban conditions, hovering over the terrain, transportation of people and goods to hard-to-reach places, etc. Helicopters are necessary in medicine, the army, transport companies, the Ministry for Emergency Situations, the Ministry of Internal Affairs. In the general structure of the fleet of aircraft used to carry out air operations, helicopters occupy 54\%. Since this type of technology is science-intensive, to maintain in a state of readiness, operating organizations require significant funds and a competently constructed operating system.

Since the first launch of the MI-1 helicopter in 1952, there have been cardinal changes in the field of Russian helicopter construction. Despite the fact that work is now actively being carried out to create perspective rotorcraft, research and development work are carried out, a large proportion of all helicopter engineering activities are still aimed at modernizing existing models and types of equipment.

Moral and physical wear and tear is inherent in the Russian fleet of vessels, besides flight characteristics and consumer properties do not correspond to modern market requirements. All this significantly affects to the safety of flights. The issue of establishing the resources and terms of service of civil aviation equipment and the transition from operation over the established resource to operation according to the current technical condition is also acute.[2] In the world practice these problems are solved by equipping the helicopters with modern hardware and new technical means. In addition to it fault diagnosis systems are being developed.

The presence of a defect and its development to a certain extent do not immediately lead to catastrophic consequences. This indicates the need to detect faults at a time as close to their generation as possible in order to take measures to repair or replace the faulty unit to prevent a possible accident. To solve this problem, special diagnostic systems are being developed that allow early detection of faults in units and assemblies. Their presence on the aircraft is a necessary prerequisite for the maintenance of "by state".

The sources of the initial data for the diagnostic systems are the signals carrying information about the technical state of the units and aggregates. These signals can have a different physical nature, it is important only that they directly or indirectly reflect the state of the object.

The fault diagnosis systems that are currently in use differ in the principles of operation. The most universal is vibration diagnostics. The essence of this diagnostic method consists in the periodic removal of vibration information, the determination of the values of diagnostic parameters, the comparison of current values with thresholds, and decision-making when exceeding them.

Progressive wear of the teeth of the gear wheels of the gearbox leads to a significant increase in the value of the overall vibration level. The appearance of fatigue damage on individual teeth (chipping, cleavage, crack at the base, failure) causes an increase in the values of the

\footnotetext{
* Corresponding author: katya2110m@yandex.ru
} 
total level of the harmonic components of the speed of the driving and driven gears.

Using this method, a large number of parameters can be diagnosed without resorting to dismantling the controlled object itself.

However, the vibration parameters are sensitive to the operating conditions of the equipment, which leads to the impossibility of comparing the results of measurements obtained at large time intervals.

The processes of friction and wear occurring in the units of gearboxes are accompanied by the loss of the metal of the conjugate parts in the form of wear products, which are carried away from the friction zone by the lubricating oil washing these parts. Hence a fundamentally different from the previous approach to diagnostics is appeared - diagnostics of the content of wear products in lubricating oil.

Varieties of this method for controlling the content of wear products in lubricating oil are: the method of spectral analysis of oil; Method of mass spectrometry; Ferrographic method; Radiometric method; X-ray spectral analysis of oil, etc.

In addition to these, in practice, there is used an approach to assess of the technical condition of the gearboxes, based on measuring the temperature of the oil, the increase of which indicates an increased load or an insufficient level of oil in the gearbox.

From the standpoint of the operation of engineering facilities, there is an importance of the frequency of diagnostic features in conditions of continuous operation. In such conditions, it is necessary to emphasize the significant technical advantage of the approach, which is based on precision phasechronometric measurements forming a time series reflecting the influence of the work process on the measured physical quantity-the time interval corresponding to different measurement intervals of the working cycle.[3] Variations of time intervals characterize the features of changing the parameters of the working cycle, the mathematical processing of which allows you to evaluate the effect of design changes on output parameters.

\section{The application of the phase- chronometric method to the diagnostics of gearboxes}

This method can be used to ensure the operation of the cyclic mechanisms of aviation equipment, namely, helicopter gearboxes. The diagnostic system based on the phase-chronometric method is based on measuring the kinematic parameters of the operating cycle of the mechanism with phase quantization and time sampling and is able to provide a relative measurement error of not more than $5 \cdot 10^{-4} \%$ under operating conditions.[4]

The functional diagram of the phase-chronometric system is shown in Figure 1.

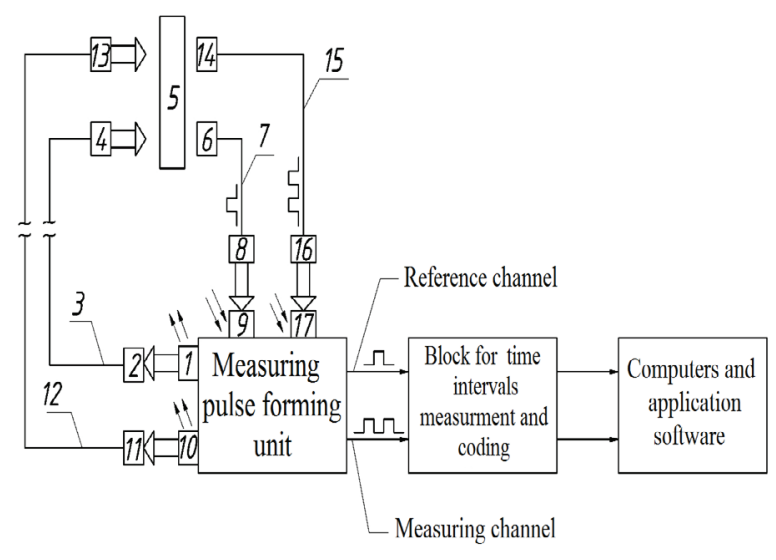

Fig. 1. Functional diagram of the phase-chronometric system.

The following designations are adopted for the reference and measuring channels, respectively: 1, 10 Radiating diode; 2, 11 - Input collimator; 3, 12 - Optical cable; 4, 13 - Output collimator; 5 - Measuring disc; 6, 14 - Input collimator; 7, 15 - Optical cable; 8, 16 Output collimator; 9, 17 - Photodiode.

The phase-chronometric system is an informationmeasuring complex. It includes an angular position sensor, a system for collecting information and determining time intervals, a personal computer, applied and special software. The system for collecting information and determining the time intervals is a board that integrally combines a highly stable pulse generator, a scale converter, one or more counters, a measuring pulse driver, and a chronocomparator.[5]

All these elements can be implemented in a microcontroller, the board of which contains an ADC.

The principle of operation of the photovoltaic system consists in the sequential recording of the duration of whole and multiple periods of output shaft rotation. The luminous flux is created by the primary photoelectric converter and the disk with grooves. The resulting light flux is transferred to the photoconverters of the electronic unit, where a measuring pulse is formed. Then the signal on the communication lines goes to the specialized computer board, where the pulses of the master quartz generator are counted. Due to it, precise measurements of the time intervals between pairs of consecutive pulses are possible. By comparing the obtained time characteristics and the results of mathematical modeling, one can judge the technical state of the diagnostic object.

\section{Analysis of data obtained by using the phase-chronometric method}

When applying the phase-chronometric method, the results of measurements are arrays of experimental data from successive time series that reflect the effect of the operation of the mechanism on time intervals. Mathematical processing of experimental data, for example, spectral analysis, allows for a general and element-by-element analysis of the operation of the mechanism. 
To make phase-chronometric diagnostics, it is necessary, first of all, to analyze and implement the work cycle of the product. After this, in detail consider the dynamics and kinematics of the interaction of details and parts of the product to form a mathematical model.

A consistent technique for processing the already acquired measurement information consists of several stages, the first of which is the acquisition and analysis of primary information in the form of rotation chronograms (mathematical modeling). Figure 2 shows the chronogram of the output shaft rotation of the gearbox at a frequency of $7600 \mathrm{rpm}$.

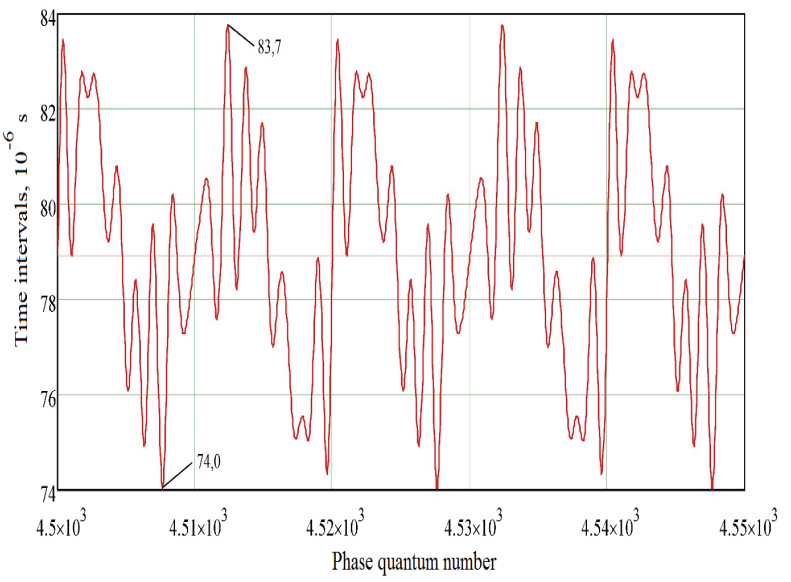

Fig. 2. Chronogram of rotation of the output shaft at $7600 \mathrm{rpm}$.

The spectral analysis (Fig. 3) showed that the natural frequencies of the mechanism correspond to $50.635 \mathrm{~Hz}$; $163.384 \mathrm{~Hz} ; 382.159 \mathrm{~Hz} ; 658.614 \mathrm{~Hz} ; 988.827 \mathrm{~Hz}$; 1004.601 Hz.

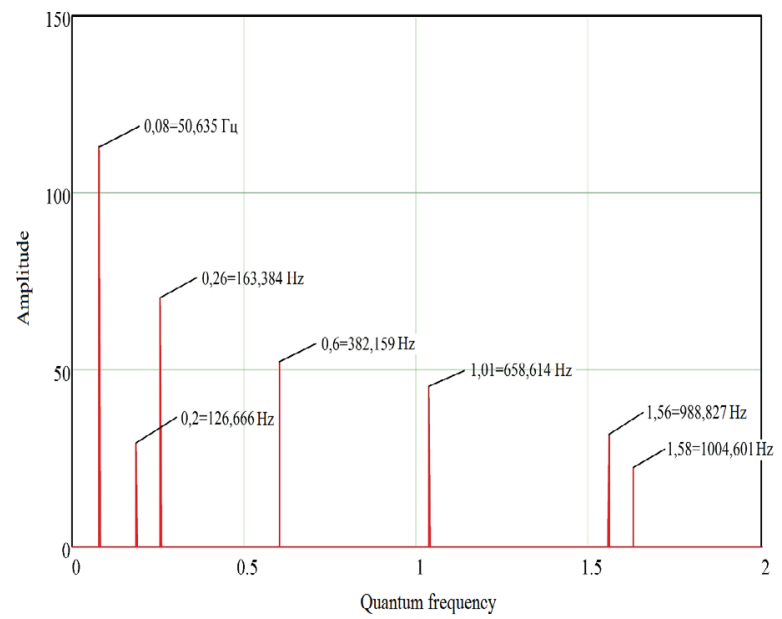

Fig. 3. Spectral analysis of the signal at $7600 \mathrm{rpm}$.

In the real process of operation and operation, there are effects associated with vibrations in the mechanism, with regime parameters, with uneven engine operation, the effect of assembly errors, temperature deformations, and the degradation of the properties of structural materials and the imbalance of the system resulting from the operation of the mechanism. All these components affect the real chronogram of the output shaft rotation. The real chronogram is shown in Figure 4.

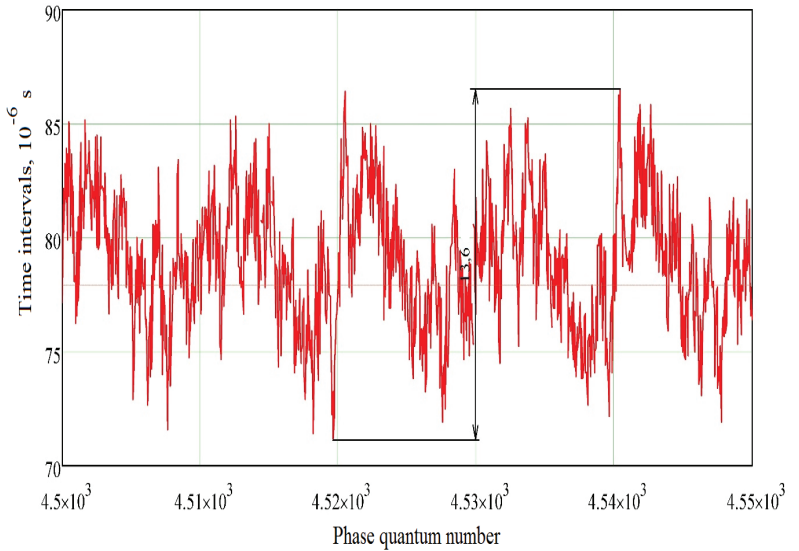

Fig. 4. Real chronogram of rotation of the output shaft at a frequency of $7600 \mathrm{rpm}$.

The spectrum of the real signal is shown in Figure 5.

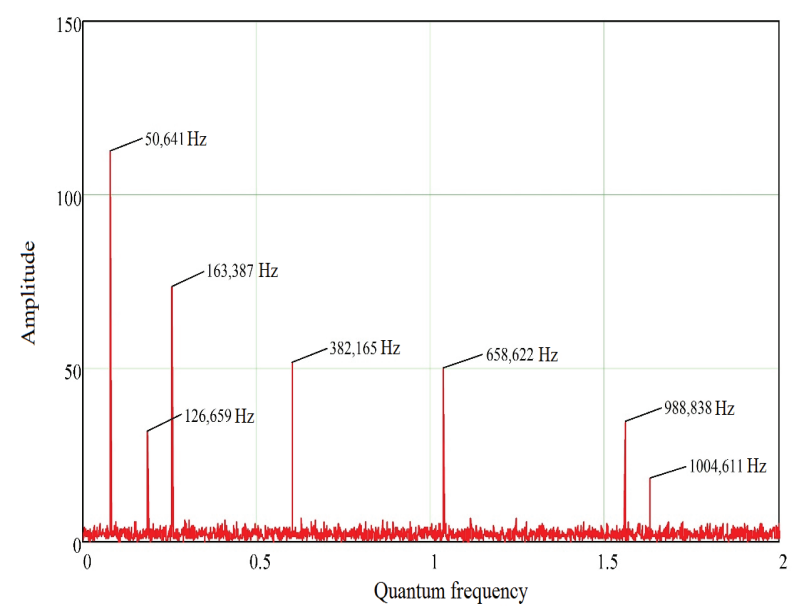

Fig. 5. Spectrum of real signal.

Comparison of the values of the natural frequencies of the mechanism, obtained as a result of mathematical and experimental modeling, shows that during operation, there is the change of own frequencies of the mechanism in comparison with the initial state. By these changes, one can judge the state of the mechanism itself. A comparison of these values is presented in Table 1.

Table 1. Comparison of the values of the natural frequencies of the mechanism.

\begin{tabular}{|c|c|c|}
\hline $\begin{array}{c}\text { The value of the natural frequencies of } \\
\text { the mechanism, Hz }\end{array}$ & $\begin{array}{c}\text { Relative error } \\
\text { of natural } \\
\text { frequencies, } \\
\Delta \varpi\end{array}$ \\
\cline { 1 - 2 } $\begin{array}{c}\text { The result of } \\
\text { mathematical } \\
\text { modeling }\end{array}$ & $\begin{array}{c}\text { The result of } \\
\text { processing the } \\
\text { experimental data }\end{array}$ & $3,8.10^{-2} \%$ \\
\hline 36,682 & 36,696 & $1,1.10^{-2 \%} \%$ \\
\hline 91,603 & 91,613 & $6,8.10^{-3} \%$ \\
\hline 218,430 & 218,445 & \multicolumn{2}{|c}{} \\
\hline
\end{tabular}


Table 1. Continued.

\begin{tabular}{|c|c|c|}
\hline \multicolumn{2}{|c|}{$\begin{array}{c}\text { The value of the natural frequencies of } \\
\text { the mechanism, Hz }\end{array}$} & $\begin{array}{c}\text { Relative error } \\
\text { of natural } \\
\text { frequencies, } \\
\Delta \varpi\end{array}$ \\
\hline $\begin{array}{c}\text { The result of } \\
\text { mathematical } \\
\text { modeling }\end{array}$ & $\begin{array}{c}\text { The result of } \\
\text { processing the } \\
\text { experimental data }\end{array}$ & \\
\hline 476,671 & 476,684 & $2,7.10^{-3} \%$ \\
\hline 608,511 & 608,519 & $1,3.10^{-3} \%$ \\
\hline 715,530 & - & - \\
\hline
\end{tabular}

Experimental studies on operating technical objects have shown that precise chronometric control of the working cycle phase ensures the detection of qualitative repetitive characteristics and individual quantitative parameters of the object under study.[6] All this testifies to the advisability of using measuring phasechrochronometric systems in the conditions of production of cyclic aggregates of aviation equipment, which will make it possible to obtain measurement information on a fundamentally new level and in the future will allow to go from operation to a specified resource for operation in accordance with technical condition.

\section{References}

1. M.I. Kiselev, V.I. Pronyakin, Y.V. Chivilev, Mir Izm. 4 (2007)

2. E.D. Metelkina, IIKYSS (2016)

3. A.S. Komshin, Stand.\& Qual. 12 (2015)

4. M.I. Kiselev, Stand.\& Qual. 10 (2013)

5. K.G. Potapov, Research and development of a method and means for assessing the current technical condition of the main drives of turning equipment based on the phase-chronometric approach (BMSTU, 2015)

6. M.I. Kiselev [and others], High Techn. 9 (2016) 